\title{
Zur Entgrenzung der Polizei - eine juridische und materialistische Kritik polizeilicher Gewalt ${ }^{* 1}$
}

Summary

For some time now, misconduct by police is discussed increasingly in Germany. The spectrum ranges from violent police actions at meetings (Stuttgart21, Blockupy) including illegal checks and inspections based on racist criteria (racial profiling) to physical attacks on persons during everyday police checks. In response to these cases, for example, the state of Berlin has introduced compulsory individual labelling of police officers so that those concerned can take legal action against individual police actions. This focus on individual misconduct causes that the perspective on a more fundamental criticism of the police are lost out of sight. Does it not cause a problem to democratically organized societies when a state apparatus holds the exclusive violence monopoly? Do the police follow only the requirements of the legislator or do they also pursue own interests in their work? The contribution attempts to approach these questions from the perspective of political theory. It will fathom to what extent the police act as an independent political actor, who becomes independent again and again from its parent act. Three astounding examples of the current debate concerning policing strategies are highlighted to explain this specific autonomisation of the police: the 'danger-areas' in Hamburg, police strategies against the Blockupy protests, as well as police treatment of football fans.

\section{Résumé}

Les écarts de conduite de la police font de plus en plus débat ces derniers temps en Allemagne. Ceux-ci couvrent tant ses interventions violentes lors de rassemblements (Stuttgart21, Blockupy) que des contrôles illégaux fondés sur des critères racistes (le racial profiling) ou des agressions physiques à l'encontre d'individus dans le cadre de contrôles de routine. En réaction à ces abus, le Land de Berlin a par exemple instauré une obligation d'identification personnelle pour chaque agent de police, de manière à permettre aux personnes concernées d'intenter une action contre des agissements policiers individuels. Cette focalisation sur les abus individuels fait toutefois totalement perdre de vue la nécessité d'une critique plus approfondie de la police. N'est-il pas problématique, dans une société démocratique, qu'un appareil d'État détienne le seul monopole du recours à la violence? La police se contente-t-elle de suivre les prescriptions du législateur ou obéit-elle aussi à ses propres intérêts dans l'exercice de son

* Maximilian Pichl ist wissenschaftliche Hilfskraft am Institut für Öffentliches Recht der GoetheUniversität, Prof. em. Frankenberg. Er hat Rechtswissenschaften und Politikwissenschaften studiert und ist Redaktionsmitglied der Zeitschrift Forum Recht.

1 Ich danke Moritz Assall, Timo Tohidipur und Marie-Luise Wallis für wertvolle Kommentare. 
travail? L'article tente de répondre à ces questions à partir d'une perspective théorique d'État. Il analyse l'ampleur de la prise d'indépendance de la police, en tant qu'acteur politique autonome, par rapport à ses bases d'habilitation légales. Cette autonomisation spécifique de la police est illustrée à l'aide de trois exemples éloquents issus des débats actuels autour des actions policières: les 'zones à risques' de Hambourg, les stratégies policières contre les protestations de Blockupy et le rapport entre la police et les supporters de football.

\section{A. Prolog}

„Als Vollstrecker einer unbeliebten Politik oder sogar als Gewalttäter, so erscheint die Polizei derzeit oft im öffentlichen Licht", stellt ein Artikel in der ZEIT fest. ${ }^{2}$ Verschiedene Ereignisse jüngerer Polizeirepression werden aufgezählt, darunter die polizeiliche Belagerung der von Flüchtlingen besetzten Gerhart-Hauptmann Schule in Berlin, der Wasserwerfereinsatz gegen protestierende Stuttgart 21-Gegner_innen sowie der Tod des Asylbewerbers Oury Jallouh in Dessau. Das Vertrauen der Menschen in die Polizei sei aufgrund dieser Gewaltexzesse und Rechtsübertretungen erschüttert. Als Forderungen an eine rechtsstaatliche Polizeiarbeit werden die Einführung einer bundesweiten individuellen Kennzeichnungspflicht von Polizist_innen und eine unabhängige Beschwerdestelle genannt, um juristisch gegen die „Täter_innen in Uniform“ vorgehen zu können. ${ }^{3}$

Diese Analyse in der ZEIT steht stellvertretend für die Debatte über Polizeigewalt in Deutschland. Es entsteht der Eindruck, dass das Hauptproblem rechtswidriger Polizeigewalt in dem Verhalten von einzelnen Beamt innen gesehen wird. Strukturelle Probleme innerhalb der Polizei werden in der Öffentlichkeit vorrangig als Ausdruck einer intransparenten Arbeit, folglich als „Kommunikationsproblem“ dargestellt. Die Polizei müsse nur an die ,juristische Kandare“ genommen werden. Eine radikale Kritik, die polizeiliche Gewalt und die Missachtung verfassungsrechtlicher Prinzipien in dem Apparat der Polizei selbst verortet oder die Legitimation der Polizei grundsätzlich in Frage stellt, kommt kaum zur Sprache.

Mit dem folgenden Beitrag möchte ich beide Formen der Polizeikritik anhand frappanter Ereignisse polizeilicher Verselbstständigung diskutieren. Die Repressionen gegen die Blockupy-Demonstrationen in Frankfurt am Main, die Ausrufung des Gefahrengebiets in Hamburg-Altona sowie der Umgang der Polizei mit Fußballfans im und um das Stadion, verweisen auf die Unmöglichkeit polizeiliche Maßnahmen rechtsstaatlich einzuhegen.

Zunächst werden die drei genannten Beispiele polizeilicher Repression dahingehend untersucht, inwieweit die Polizei geltendes Recht missachtet hat. Diese Kritik bewegt sich innerhalb der Voraussetzungen des Rechts. Ihr liegt ein verfassungsrechtlicher Maßstab zugrunde, der polizeiliche Maßnahmen, ihre gesetzlichen Grundlagen und die hierzu ergangene Rechtsprechung daraufhin untersucht, ob grundrechtlich verbürgte

2 Thurm, Polizeigewalt: Die Polizei, dein Freund und Helfer, in: Die Zeit vom 7. Juli 2014.

3 Vgl. ebenfalls: Amnesty International, Täter unbekannt -Mangelnde Aufklärung von mutmaßlichen Misshandlungen durch die Polizei, Berlin 2010. 
Freiheiten ermöglicht oder beschränkt werden. Die juridische Kritik hat aber Grenzen. Sie vermag oft nicht zu erklären, warum es in der Praxis zu einem „Auseinanderklaffen von Verfassungsnorm und Verfassungswirklichkeit, von Proklamation und Exekuti$o n^{* 4}$ kommt. Denn ihr liegt ein Verständnis zugrunde, dass die Polizei als exekutiver Staatsapparat an das Recht gebunden ist (Art. 20 III GG) und somit rechtliche Verfahrensweisen nicht nur zu befolgen hat, sondern mit hervorbringen muss. Die Polizei wird nur als ausführendes Organ des Rechts wahrgenommen. Ich möchte die juridische Kritik deshalb um eine staatstheoretische Kritik ergänzen und schließe hierbei insbesondere an Debatten der materialistischen Staatstheorie an. ${ }^{5}$ Sie greift den Widerspruch zwischen Verfassungsnorm und Verfassungswirklichkeit als kennzeichnendes Prinzip des Verhältnisses zwischen Recht und Polizei auf. Ausgehend von diesem Widerspruch wird die Frage gestellt, ob es ausreicht, dem Polizeiapparat mit einer juridischen Kritik zu begegnen oder ob sich die Polizei nicht stets von ihren Ermächtigungsgrundlagen verselbstständigen kann. Die Polizei wird mit dieser Kritik nicht allein als Vollzugsinstrument des Staates verstanden, sondern als eine aus den gesellschaftlichen Verhältnissen hervorgegangene Institution, in der sich eigenständige politische Interessen und Strategien verfestigen, die Polizei folglich selbst zum politischen Akteur wird.

\section{B. Zur juridischen Kritik polizeilicher Repression}

\section{Blockupy-Aktionstage}

„Das Recht des Bürgers, durch Ausübung der Versammlungsfreiheit aktiv am politischen Meinungsbildungsprozeß und Willensbildungsprozeß teilzunehmen, gehört zu den unentbehrlichen Funktionselementen eines demokratischen Gemeinwesens. "6 Mit seiner Brokdorf-Entscheidung hat das Bundesverfassungsgericht 1985 das Versammlungsrecht aus Art. 8 Abs. 1 GG zu einem konstitutiven Merkmal der Demokratie erklärt. Selten hat das Gericht Freiheitsrechte der Bürger_innen in dieser Deutlichkeit mit juristischer Vehemenz ausbuchstabiert und dem Staat strenge Vorgaben für die Einschränkbarkeit der Versammlungsfreiheit gesetzt. Die Blockupy-Aktionstage im Frankfurter Bankenviertel sind zu einem Prüfstein geworden, inwieweit dieser rechtsstaatliche Maßstab Geltung beansprucht.

\section{Versammlungsfreie Räume}

Das Blockupy-Bündnis, bestehend aus außerparlamentarischen linken Gruppen, Gewerkschaften, Parteien und Bürger_innenrechtsorganisationen, rief 2012 und 2013 in Frankfurt am Main zu politischen Aktionen gegen die Austeritätspolitik in der Finanzkrise auf. Man bediente sich klassischer Demonstrationen und Kundgebungen, nutzte

4 Agnoli, Von der kritischen Politologie zur Kritik der Politik, in: ders., Die Transformation der Demokratie und verwandte Schriften, Hamburg 2004, 196.

5 Hierbei konzentriere ich mich auf Vertreter_innen der Staatsableitungsdebatte und der neueren Forschung zu der Theorie von Nicos Poulantzas.

6 Vgl. BVerfGE 69, $315 \mathrm{ff}$. 
aber auch Formen zivilen Ungehorsams wie Platzbesetzungen im Frankfurter Bankenviertel oder Blockaden am Frankfurter Flughafen. In beiden Jahren verfolgten sowohl das Land Hessen, das Frankfurter Ordnungsamt als auch die Frankfurter Polizei einen grundrechtsfeindlichen Umgang mit den Protesten. Bereits vor den Protesttagen 2012 beschworen der Ordnungsdezernent Markus Frank (CDU) und die Polizei ein Schreckenszenario gewalttätiger Proteste in der Öffentlichkeit, um die Aktionstage diskursiv zu delegitimieren. ${ }^{7}$

Das Ordnungsdezernat setzte ein Totalverbot sämtlicher Versammlungen während der Aktionstage durch. ${ }^{8}$ In seiner Brokdorf-Entscheidung hatte das Bundesverfassungsgericht noch deutlich hervorgehoben, dass ein „Verbot nur zum Schutz gleichwertiger Rechtsgüter unter strikter Wahrung des Grundsatzes der Verhältnismäßigkeit und nur bei einer unmittelbaren, aus erkennbaren Umständen herleitbaren Gefährdung dieser Rechtsgüter erfolgen" dürfe. ${ }^{9}$ Ein Versammlungsverbot stellt folglich immer ein ultima ratio Mittel des Staates dar. Die Polizei Frankfurt und das Ordnungsdezernat begründeten diesen Bruch mit der Rechtsprechung des Bundesverfassungsgerichts mit einer Gefahrenprognose, die sie aus dem Verlauf der zwei Monate vorher stattgefundenen linksradikalen Demonstration M31 ableiteten, bei der es zu Sachbeschädigungen in der Frankfurter Innenstadt und zu einem Polizeikessel gekommen war. Nach den Vorgaben des Bundesverfassungsgerichts hätte die Polizei aber während der konkreten BlockupyAktionstage nachweisen müssen, dass die Demonstrationen einen gewalttätigen Verlauf nehmen könnten. Mehrere Wochen vorher war diese Gefahrenprognose nicht zu rechtfertigen. Im Übrigen bewertete das Ordnungsdezernat die Demonstrationen und Blockaden des Blockupy-Bündnisses als „schwerwiegende Nachteile“ für die Frankfurter Einwohner und Geschäftsansässige sowie Reisende und allen übrigen Menschen, ${ }^{10}$ respektive ihrer Grundrechte aus Art. 2, 4, 12 und 14 GG. Das Komitee für Grundrechte und Demokratie bemerkt in seiner Demonstrationsbeobachtung zutreffend, dass ,der ungestörte Alltag der Käufer und Käuferinnen, die Interessen der Geschäftsleute an ungehindertem Gewinnstreben und die Arbeit der Banken zu Grundrechten aufgewertet werden, die angeblich ein Außerkraftsetzen des Grundrechts auf Versammlungsfreiheit gewährleisten."11 Dabei hatte das Bundesverfassungsgericht erst 2011 in seiner Fraport-Entscheidung erklärt, dass „ein vom Elend der Welt unbeschwertes Gemüt des

7 Vgl. die Aussagen des Ordnungsdezernenten Markus Frank, in: Stadt Frankfurt am Main: Wortprotokoll über die 11. Plenarsitzung der Stadtverordnetenversammlung am Donnerstag, den 03. Mai 2012, Frankfurt am Main; siehe für eine Übersicht des Diskurses um die Aktionstage, Pichl, Normalisierung des Ausnahmezustands - eine Rückschau auf die Blockupy Aktionstage in Frankfurt am Main, Juridikum, 03/2012, S. 345 ff. (346 f.).

8 Zusätzlich erhielten über 400 Personen gestützt auf $\S 31$ Abs. 3 HSOG Aufenthaltsverbote für die gesamte Innenstadt Frankfurt am Main. Hiergegen erwirkten die Betroffenen erfolgreich einstweiligen Rechtsschutz und bekamen jeweils 500 Euro Schadensersatz zugesprochen. Die Aufenthaltsverbote waren rechtswidrig, diese nur zur Verhinderung von Straftaten erlaubt sind, die Absicht an verbotenen Versammlungen teilzunehmen aber lediglich eine Ordnungswidrigkeit darstellt, vgl. Stolle/Voigt, Versammlungsvermeidungsstrategien. Behörden und Justiz gegen die „Blockupy“-Proteste in Frankfurt am Main, KJ 02/2014, $186 \mathrm{f}$.

9 Vgl. BVerfGE 69, $315 \mathrm{ff}$.

10 Zitat aus der Verbotsverfügung der Stadt Frankfurt, zitiert nach: Komitee für Grundrechte und Demokratie (Hrsg.): Blockupy 2013. Der Polizeikessel am 1. Juni 2013, Köln 2013, 14.

11 Ebd., 15. 
Bürger kein Belang (ist), zu dessen Schutz der Staat Grundrechtspositionen einschränken darf."12 Versammlungen sind im demokratischen Rechtsstaat eine Form der Machtkritik. Der Staat und die sich von der Versammlung gestört fühlenden Bürger_innen haben auch unliebsame Demonstrationsmittel zu ertragen.

Diesen Maßstab der Rechtsprechung haben der Hessische Verwaltungsgerichtshof ${ }^{13}$ und überraschenderweise auch das Bundesverfassungsgericht selbst, das über die Reichweite seiner Entscheidungen scheinbar oft genug erstaunt ist, ${ }^{14}$ nicht eingehalten und alle Versammlungsverbote bestätigt, mit Ausnahme der Großdemonstration - die mit 25.000 Teilnehmer_innen friedlich verlaufen ist.

\section{Der Polizeikessel am 1. Juni 2013}

Trotz der massiven Repression wiederholten sich die Blockupy-Aktionstage 2013 in Frankfurt am Main. Zu der - auch von den Gerichten erlaubten -Abschlussdemonstration am 1. Juni 2013 waren 10.000 Demonstrant_innen gekommen. Nach nur 30 Minuten wurde der vordere Teil der Demonstration von der Polizei umzingelt. ${ }^{15}$ Die Polizei begründete ihre Maßnahme damit, in den Reihen des „schwarzen Blocks“ seien Ordnungswidrigkeiten und Straftaten begangen worden, obschon externe Beobachter wie das Komitee für Grundrechte und Demokratie bis dahin von einer vollkommen friedlichen Demonstration sprechen. ${ }^{16}$ Knapp 1.000 Demonstrant_innen wurden über acht Stunden eingekesselt. Aufgrund von rabiat geführten Kontrollen der Personalien kam es dazu, dass einzelne Demonstrant_innen bewusstlos am Boden liegen blieben, darunter auch ein Pressefotograf. Der hintere, nicht eingekesselte Teil der Demonstration wurde von der Polizei aufgefordert, ohne die eingekesselten Demonstrant_innen weiterzuziehen. Als die restlichen 9.000 Menschen dem nicht Folge leisteten, versuchte die Polizei mit Pfefferspray die Menge auseinander zu treiben. Erst am späten Abend wurde der Kessel aufgelöst, die Demonstration konnte ihr Ziel, die Europäische Zentralbank, nicht mehr erreichen.

Es sei noch einmal an das Bundesverfassungsgericht erinnert: „Steht nicht zu befürchten, daß eine Demonstration im ganzen einen unfriedlichen Verlauf nimmt oder daß der Veranstalter und sein Anhang einen solchen Verlauf anstreben oder zumindest billigen, bleibt für die friedlichen Teilnehmer der von der Verfassung jedem Staatsbür-

12 BVerfGE 128, 226, $266(22.2 .2011)$.

13 HessVGH, B. v. 16.5.2012 - 8 B 1158/12.

14 Oliver Lepsius bemerkt hierzu, dass dieses Problem in der Methodik des BVerfG selbst begründet liegt. Das BVerfG formuliert im Hinblick auf konkrete Rechtsfragen allgemeine Maßstäbe, deren beschauliche Rechtsfolgen zu einem Auseinanderklaffen zwischen Theorie und Praxis führen: „Der politische Prozess wird mit Rationalitätserwartungen belastet, die quer zu seinen Funktionsbedingungen verlaufen und seine Handlungsfähigkeit nicht stärken.“ (vgl. Lepsius, Die maßstabssetzende Gewalt, 2011, in: Jestaedt, u.a. (Hrsg.): Das entgrenzte Gericht. Eine kritische Bilanz nach sechzig Jahren Bundesverfassungsgericht, Frankfurt am Main 2011, $159 \mathrm{ff}$. (212).

15 Ein detaillierter Bericht zu der Demonstration und dem Polizeieinsatz findet sich bei Komitee: (Fn. 10), 36ff.

16 Vgl. Komitee, (Fn. 10), 37ff. 
ger garantierte Schutz der Versammlungsfreiheit auch dann erhalten, wenn mit Ausschreitungen durch einzelne oder einer Minderheit zu rechnen ist. "17

Die Versammlungsfreiheit Vieler kann nicht durch gewalttätige Aktionen Einzelner beschränkt werden. Das Brokdorf-Urteil wird durch die Polizei und die Ordnungsämter versammlungsfeindlich ausgelegt. Ein ,unfriedlicher Verlauf“ wurde bei Blockupy alleine durch die Verwendung von möglichen Vermummungsgegenständen angenommen, mithin Regenschirme, die die Demonstrant_innen bei sich trugen. Dabei dürfen an die Friedlichkeit von Versammlungen keine „übersteigerten Anforderungen“ gestellt werden, insbesondere eine „Vermummung oder das Tragen von sog. Schutzwaffen allein genügt dafür nicht. " ${ }^{18}$ Entgegen der Gerichtsentscheidung hielt die Polizei an ihrer Gefahrenprognose fest. Und das obwohl die Demonstration aus dem Jahr zuvor mit mehr Teilnehmer_innen friedlich verlaufen war. Deshalb wurde in der Presse darüber diskutiert, ob der Kessel nicht von Anfang an politisch motiviert bzw. eine ,vorbereitete Falle" gewesen sei. ${ }^{19}$

\section{Versagen der Judikative}

Die versammlungsfeindlichen Maßnahmen der Polizei unterlagen während der Demonstration praktisch keiner gerichtlichen Kontrolle. Verschiedene Anwält_innen hatten bereits im Laufe der Kesselung Freilassungsanträge beim richterlichen Notdienst gestellt. Die zuständige Richterin sah sich als nicht zuständig an, traf keine Entscheidung und verabschiedete sich, noch während der Kessel bestand, in den Feierabend. ${ }^{20}$ Die Judikative hat bei Blockupy ihre originäre Aufgabe nicht wahrgenommen: Gerade bei einer Großdemonstration, die in der Öffentlichkeit über mehrere Monate diskutiert wurde, müssen die Gerichte nach Art. 19 Abs. 4 GG einen effektiven Rechtsschutz zur Verfügung stellen, der polizeiliche Maßnahmen auf ihre Verhältnismäßigkeit überprüft und Freiheitsrechte garantiert. Es reicht nicht aus, dass Gerichte immer erst im Nachgang einer Demonstration ihre Kontrolltätigkeit aufnehmen. Die Gefährdung von Grundrechten durch den Staat ist unmittelbar zu verhindern.

Das juristische Nachspiel um Blockupy dauert an. Aufgrund der hessischen Landtagswahlen im September 2013 ist es nicht zu der Einsetzung eines Untersuchungsausschusses gekommen, der die Intentionen der Polizei und des Innenministeriums offenlegen könnte. Das Verwaltungsgericht Frankfurt am Main hat zwischenzeitlich den Kessel als rechtmäßig beurteilt, die Kläger_innen haben hiergegen Berufung vor dem Hessischen Verwaltungsgerichtshof beantragt. Das Verwaltungsgericht befand das äußere Erscheinungsbild der Demonstration hätte einen Eingriff gerechtfertigt. ${ }^{21}$ Inwiefern andere Gerichte diese Abweichung vom Brokdorf-Urteil stützen, bleibt abzuwarten.

17 Vgl. BVerfGE 69, $315 \mathrm{ff}$.

18 Vgl. Schneider, in: Epping/Hillgruber, GG-Kommentar, Art. 8 GG, Rn. 13 ff.

19 Vgl. Frankfurter Rundschau vom 6. Juni 2013; Süddeutsche Zeitung vom 2. Juni 2013.

20 Vgl. Stolle/Voigt, (Fn. 8) 192; Komitee, (Fn. 10) 63.

21 Vgl. VG Frankfurt am Main, 5 K 2340/13.F; 5 K 2334/13.F. 


\section{Gefahrengebiet in Hamburg}

Das zweite Beispiel polizeilicher Repressionsmaßnahmen ereignete sich Anfang 2014 in Hamburg. Verschiedene soziale Konflikte in der Stadt hatten sich zugespitzt: das seit 20 Jahren von Linksautonomen besetzte Haus Rote Flora im Schanzenviertel wurde mit einer Räumung durch den Eigentümer bedroht; der Hamburger Senat führte rigide Kontrollen bei der Gruppe der Lampedusa-Flüchtlinge durch, die in einer Kirche in St. Pauli Zuflucht gesucht hatten; zudem stand die Räumung der Esso-Häuser bevor, die einen zentralen Anlaufpunkt des Kiezes darstellen. Es kam zu Auseinandersetzungen zwischen der Polizei und linksradikalen Gruppierungen, beispielsweise im Zuge einer Demonstration für den Erhalt der Roten Flora. Als am 29. Dezember 2013 die Polizeistation Davidwache im Schanzenviertel von Personen, angeblich aus der linken Szene, angegriffen und ein Polizeibeamter schwer verletzt wurde, richtete die Hamburger Polizei im Bezirk Altona ein sogenanntes Gefahrengebiet ein. Ziel sollte es sein, Personen aus der linken Szene zu kontrollieren, um Gegenstände wie Böller, Pfefferspray oder sonstige Utensilien sicherzustellen. Ob der Angriff auf die Davidwache tatsächlich politisch motiviert war oder das Resultat eines, ,üblichen“ Konflikts auf der Reeperbahn darstellte, ist bis heute ungeklärt. Viele Anzeichen sprechen jedoch dafür, dass die Polizei der Öffentlichkeit falsche Informationen offenbart hat. ${ }^{22}$

\section{Zur Funktion der Gefahrengebiete}

Unter der Regierung von CDU und Schill-Partei novellierte der Hamburger Senat 2005 den $\S 4$ Abs. 2 S. 1 des Gesetzes über die Datenverarbeitung der Polizei (PolDVG), in dem es heißt:

„Die Polizei darf im öffentlichen Raum in einem bestimmten Gebiet Personen kurzfristig anhalten, befragen, ihre Identität feststellen und mitgeführte Sachen in Augenschein nehmen, soweit aufgrund von konkreten Lageerkenntnissen anzunehmen ist, dass in diesem Gebiet Straftaten von erheblicher Bedeutung begangen werden und die Maßnahme zur Verhütung von Straftaten erforderlich ist."

Die Entscheidung, ob ein Gefahrengebiet eingerichtet wird oder nicht, liegt demnach nicht in der Zuständigkeit des Gesetzgebers, sondern die Polizei - konkreter: die in den Bezirken tätigen Revierleiter - urteilt aufgrund ihrer Lageerkenntnisse. In Hamburg wurden bereits über 40 Gefahrengebiete eingerichtet, manche von kurzer Dauer, andere bestehen ständig, wie beispielsweise um die Hamburger Reeperbahn. Die Gefahrengebiete wurden aufgrund von Drogenkriminalität, links orientierten politischen Demonstrationen, bei Fußballspielen oder Gewaltdelikten ausgerufen. ${ }^{23}$

Durch die Gefahrengebiete kann die Polizei Personen in den entsprechenden Gebieten kontrollieren, ohne dass diese mit ihrem Verhalten einen konkreten Anlass begründet hätten - alleine der Aufenthalt in dem Gebiet, mithin eine abstrakte Gefahr, genügt für

22 Vgl. Reisin,/Gensing, Anschlag auf Davidwache. Was bislang bekannt ist, auf Publikative 2014, http://publikative.org/2014/01/06/anschlag-auf-davidwache-was-bislang bekannt-ist/ (zuletzt aufgerufen am 25.7.2014).

23 Vgl. Belina/Wehrheim, Gefahrengebiete. Durch die Abstraktion des Sozialen zur Reproduktion gesellschaftlicher Strukturen, Soziale Probleme 02/2011, 207 ff. (219). 
polizeiliche Maßnahmen aus. Im Hamburger Stadtteil St. Georg, der als „Problembezirk“ gilt, besteht ein immerwährendes Gefahrengebiet, das Moritz Assall als ,raumbezogenes Mittel zur stadtpolitischen Reinigung der als 'schmuddelig' und unrepräsentativ identifizierten Gegend um den Hauptbahnhof" versteht. ${ }^{24}$

\section{Das Gefahrengebiet in Altona 2014}

Das Gefahrengebiet in Altona 2014 stellte das bisher flächenmäßig größte Gefahrengebiet dar, das den Wohnort von 80.000 Personen umfasste. Das am 04. Januar 2014 auf unbestimmte Zeit eingerichtete Gebiet wurde nach massiven öffentlichen Protesten am 09. Januar auf sogenannte „Gefahreninseln“ im Umkreis dreier Polizeikommissariate verkleinert. In den fünf dazwischenliegenden Tagen hat die Polizei 756 Personen angehalten, 22 Strafanzeigen angefertigt, in 209 Fällen mitgeführte Sachen in Augenschein genommen - dabei erlangte eine konfiszierte Klobürste bundesweite Berühmtheit $^{25}$ - sowie in 172 Fällen Aufenthaltsverbote ausgesprochen, in 12 Fällen Platzverweise erteilt und 65 Personen in Gewahrsam genommen. ${ }^{26}$

Das Altonaer Gefahrengebiet verdeutlicht die Problematik des $\S 4$ Abs. 2 S. 1 PolDVG. Zunächst ist beachtlich, dass das Wort „Gefahrengebiet“ in der polizeilichen Ermächtigungsgrundlage nicht auftaucht. Es gibt auch keine Hinweise auf räumliche oder zeitliche Beschränkungen. Ginge man alleine vom Wortlaut aus, könnte die Hamburger Polizei praktisch die gesamte Stadt zum Gefahrengebiet erklären. Auch die in der Ermächtigungsgrundlage genannten Maßnahmen wie Anhalten, Befragen, Identitätsfeststellungen oder Inaugenscheinnahmen, sind bereits in den Hamburger Polizeigesetzen geregelt. ${ }^{27}$ Nassim Madjidian erinnert daran, dass $\S 4$ Abs. 2 S. 1 PolDVG seinerzeit eingeführt wurde, um gegen die im Umfeld des Hamburger Hafens tätige organisierte Kriminalität, sowie gegen Menschen- und Waffenhandel vorzugehen. ${ }^{28}$ Die tatsächliche Praxis der Polizei zeigt aber, dass diese Ermächtigungsgrundlage auf alle möglichen Handlungsfelder angewendet wird. Inwieweit $\S 4$ Abs. 2 S. 1 PolDVG deshalb überhaupt mit dem Bestimmtheitsgebot nach Art. 20 Abs. 3 GG vereinbar ist, ist fraglich. ${ }^{29}$ Hiermit hatte sich das Verwaltungsgericht Hamburg auseinanderzusetzen, nachdem eine Anwohnerin des Schanzenviertels 2012 geklagt hatte. Das Verwaltungsgericht erkannte zwar, dass die Ermächtigungsgrundlage in Grundrechte der Betroffenen eingreift, ermöglichte aber die Verfassungskonformität. Der Begriff „,bestimmte Gebiete" in der Norm verweise darauf, dass nicht das gesamte Stadtgebiet betroffen sein kann. ${ }^{30} \mathrm{Ob}$ das Gericht auch hinsichtlich eines derart großen Gebietes wie in Altona weiterhin von seiner Rechtsprechung überzeugt wäre, bleibt dahingestellt.

24 Vgl. Assall, Im stillen Reich der Polizei. Verselbstständigungen der Polizei im Gefahrengebiet und Drumherum, Forum Recht 03/2014, 77 ff. (78).

25 Vgl. Wierich, Die Wirkungsmacht der Klobürste, Freitag 11.1.2014.

26 Vgl. http://www.hamburg.de/innenbehoerde/4251394/2014-01-15-bis-bt-hintergrundinfo gefahrengebiet.html, (zuletzt aufgerufen am 25.7.2014).

27 Vgl. Madjidian, Gefahrengebiet. Polizei außer Kontrolle?, Forum Recht 03/2014, 80 f. (80).

28 Ebd., 80.

29 Vgl. Ernst, Anlassunabhängige Personenkontrollen und Gefahrengebiete, NVwZ 2014, 635; BVerfGE 100, 313, 359.

30 Vgl. VG Hamburg, Urt. 2.10.2012 - 5 K 1236/ 11, BeckRs 2013, 52593. 
Denn die Beschränkungen von Grundrechten der betroffenen Personen in den Gefahrengebieten sind intensiv. In einem Gutachten des Hamburgischen Datenschutzbeauftragen wird darauf verwiesen, dass in das Recht der kontrollierten Personen auf informationelle Selbstbestimmung aus Art. 2 Abs. 1 i.V.m. Art. 1 Abs. 1 GG umso stärker eingegriffen werde, je weniger die Personen mit einer Kontrolle rechnen müssen. ${ }^{31}$ Selbst wenn man den Vorgaben des VG Hamburg zur Einrichtung von Gefahrengebieten folgen würde, hätte das Gefahrengebiet vom 4.1.2014 diese Voraussetzungen nicht erfüllt. Weder enthielt der Antrag der Polizei Lageerkenntnisse über die mögliche Begehung von Straftaten in dem ausgewiesenen Gebiet, ${ }^{32}$ noch wurde der Polizeichef über die Errichtung informiert. ${ }^{33}$ Bedenklich ist zudem die mit drei Monaten relativ lange anberaumte Speicherfrist der in den Gefahrengebieten gesammelten Daten. „Bei jeder Kontrolle dieser Personen in anderem Zusammenhang wird zumindest 3 Monate lang die Kontrolle im Gefahrengebiet abgerufen. Dies kann bei den kontrollierenden Polizisten zu einem gewissen Vorbehalt führen und stigmatisierende Folgen für die kontrollierten Bürger haben." 34

Eine höchstrichterliche Entscheidung über die verfassungsrechtliche Vereinbarkeit der fragwürdigen Ermächtigungsgrundlage aus dem Hamburgischen Polizeigesetz steht aus. So kann die Polizei bei Bedarf weiterhin in ganzen Stadtgebieten die Rechte von zehntausenden Bürger_innen beschränken.

\section{Repressionen gegen Fußballfans}

Als drittes und letztes Beispiel aktuell diskutierter Polizeirepression dient der Umgang mit Fußballfans. Nicht zuletzt der Polizeieinsatz beim Champions League Spiel zwischen dem FC Schalke 04 und Panathinaikos Athen am 21. August 2013 oder der Platzsturm von Düsseldorfer Fußballfans beim Aufstiegsspiel gegen Hertha BSC Berlin, haben das Fußballstadion als relevantes Betätigungsfeld der Polizei in den öffentlichen Fokus gerückt. Die bundesweite Arbeitsgemeinschaft der Fananwälte sieht in dieser Entwicklung eine Konstruktion des Fußballfans als „Feindbild Nummer 1“ und das Stadion als Laboratorium polizeilicher Repression. ${ }^{35}$ Es geht bei diesem Beispiel nicht um ein singuläres Ereignis, sondern um Schlaglichter polizeilicher Repression.

31 Vgl. Caspar/Menzel, Datenschutzrechtliche Bewertungen des polizeilichen Gefahrengebietes im Bezirk Altona vom 04.-13.1.2014. Gutachten für den Hamburgischen Beauftragten für Datenschutz und Informationsfreiheit, Hamburg 2014, 11.

32 Ebd., $16 \mathrm{ff}$.

33 Vgl. Madjidian, (Fn. 27), 81.

34 Vgl. Casper/Menzel, (Fn. 31), 7.

35 Vgl. Verleih, Feindbild Fußballfan. Strafrechtliche Repressionen in und um das Stadion. Interview mit RA Waltraut Verleih, in: Forum Recht 04/2014 (Im Erscheinen); Arbeitsgemeinschaft Fananwälte: Warum führt der Repressionsapparat seine Übungen eigentlich an Fußballfans durch?, Die Rote Hilfe 03/2014, 25ff. 


\section{Fußballfans und Gewalt}

In Deutschland wurde der Angriff deutscher Hooligans auf den französischen Polizisten Daniel Nivel im Rahmen der Fußball-Weltmeisterschaft 1998 zu einem Kristallisationspunkt der Debatte über Gewalt in und um das Stadion. Die Sicherheitsdebatte in europäischen Fußballstadien wurde insbesondere durch die Hillsborough-Katastrophe von 1989 angefacht, bei der 98 Menschen ums Leben kamen. Danach wurden die Ticketpreise in englischen Stadien hochgetrieben, Stehplätze abgeschafft und die Kameratechnologie Closed-Circuit-Television (CCTV) eingeführt. Die Polizei und die britischen Medien gaben randalierenden Fans die Schuld für die Hillsborough-Toten. Erst Jahre später kamen die tatsächlichen Untersuchungsakten an die Öffentlichkeit, mit denen bewiesen wurde, dass die Sicherheitskräfte vor Ort versagt und die Polizei 164 Aussagen von Beamt_innen manipuliert hatten. ${ }^{36}$ Der britische Premier David Cameron sowie die an der Skandalisierung maßgeblich beteiligte Boulevard-Zeitung The Sun entschuldigten sich bei den Angehörigen der Toten. Die Fußballkultur in den britischen Stadien hatte sich dennoch radikal verändert. Die organisierten Fangruppen (sogenannte Ultras) wurden aus den Stadien gedrängt, nicht freiwillig, sondern mit Zwang und Disziplinierung.

Dabei wird vergessen, dass viele Ultra-Gruppen nicht nur in sozialen Projekten engagiert sind, sondern auch die Dominanz rechter Fangruppierungen aufbrechen konnten.

\section{Repressionsmittel}

Die Polizei wendet gegen Fußballfans Repressionsmittel an, die sie einerseits im Stadion austestet und später auf andere gesellschaftliche Bereiche überträgt, andererseits werden Maßnahmen vorgenommen, die gegenüber politischen Gruppen undenkbar wären. Eine Anwältin, die seit Jahren Fußballfans juristisch verteidigt, erläutert: „Die Entrechtung Betroffener ist häufig größer, es wird weniger genau ermittelt, selten Entlastendes. Beschuldigte und Angeklagte stehen oft großen Vorurteilen und Vorbehalten gegenüber." 37

Die Gerichte trauen der Polizei zu, eine Beurteilung über eine mögliche Strafbarkeit eines Fußballfans treffen zu können. Die Polizei setzt bei Fußballspielen sogenannte Szenekundige Beamte (SKBs) ein, die Informationen über Ultra-Gruppen sammeln und Querverbindungen zwischen einzelnen Personen herstellen. Für die Gefahrenprognose, die beispielsweise bei Meldeauflagen vorausgesetzt wird, reicht bereits ein Eintrag in die Datei „Gewalttäter Sport“" aus, die das Ergebnis von den Ermittlungen der SKBs ist. Dabei sei ,mittlerweile gerichtsbekannt, dass die Pflege polizeilicher Dateien äußerst nachlässig betrieben wird. Polizeiliche Datenbestände führen sozusagen ein Eigenleben." ${ }^{38}$ Eine Konkretisierung des Tatvorwurfs bleibt aus, es gebe kein Interesse an Tataufklärung. ${ }^{39}$ Die Folge sind Aufenthaltsverbote für Fans in bestimmten Städten, Sta-

36 Vgl. http://www.hfdinfo.com, zuletzt aufgerufen am 25.7.2014.

37 Vgl. Verleih, (Fn. 35).

38 Vgl. Petersen-Thrö/Elzermann, Die Meldeauflage als probates Gefahrenabwehrmittel im Vorfeld von Veranstaltungen, KommJur 2006, 289 ff. (296).

39 Vgl. Verleih, (Fn. 35). 
dionverbote, ${ }^{40}$ Meldeauflagen oder sogar Ausreiseverbote bei internationalen Spielen. ${ }^{41}$ In einzelnen Fällen wird sogar ein Präventivgewahrsam angeordnet, sofern das internationale Ansehen der Bundesrepublik Deutschland durch Ausschreitungen von Hooligans im Ausland gefährdet sei. ${ }^{42}$ Zuletzt hat der Europäische Gerichtshof für Menschenrechte in der Rechtssache Ostendorf Vs. Deutschland den Präventivgewahrsam für vereinbar mit der EMRK erklärt. ${ }^{43}$ Der EGMR erachtete den Rechtfertigungsgrund aus Art. 5 Abs. 1 S. 2 Buchst. b EMRK für einschlägig, wonach der Freiheitsentzug zur Erzwingung einer gesetzlichen Pflicht erlaubt ist. ${ }^{44}$ Dabei bricht der EGMR mit seiner bisherigen Rechtsprechung, dass gerade keine allgemeine Pflicht zur Beachtung von Gesetzen bestehe, sondern konkrete Verpflichtungen bestehen müssten. ${ }^{45}$

Viele Fußballfans verfügen nicht über die finanziellen Mittel um alle gegen sie gerichteten Maßnahmen vor den Verwaltungsgerichten zu überprüfen. ${ }^{46}$ Grundrechtsverstöße werden deshalb zumeist nicht juristisch aufgearbeitet.

\section{Grenzen juridischer Kritik}

Die drei aufgeworfenen Beispiele haben aktuelle Ereignisse und Bereiche polizeilicher Repression einer juridischen Kritik unterzogen. In allen drei Bereichen wurde deutlich, dass die Polizei entgegen geltender Rechtsnormen handelt - und die Gerichte dies teilweise unterbinden, teilweise aber auch von bislang etablierten Rechtsprechungen abweichen, um polizeiliche Maßnahmen (nachträglich) zu legalisieren. Ein Problem der juridischen Kritik wurde offenbar: Sie ist zwar in der Lage anhand immanenter Kritikmaßstäbe verfassungsrechtliche Rechtsübertretungen festzustellen. Jedoch ist es mit einer allein an rechtlichen Kategorien orientierten Kritik nicht möglich herauszufinden, wie die Polizei überhaupt in der Lage sein kann sich von ihren Ermächtigungsgrundlagen und der Bindung an die Grundrechte (Art. 1 Abs. 3, 20 Abs. 3 GG) zu verselbstständigen. Das Recht greift erst ein, wenn ein Fall von polizeilicher Repression entstanden ist. Die juridische Kritik formuliert sich deshalb allzu häufig als reiner Appell an die Polizei, endlich die verfassungsrechtlichen Maßstäbe anzuerkennen. Polizeiliche Entgrenzungen vom Recht werden als Normabweichungen im Einzelfall gewertet und nicht als dauerhafte, strukturbedingte Verselbstständigungen. Wie es dazu kam, warum dies möglich ist, entzieht sich einer juridischen Logik; mit ihr lässt sich nicht begreifen warum die rechtliche Einhegung der Polizei immer wieder scheitert. Sie bedarf notwendig einer Ergänzung, um die Kritik an polizeilicher Verselbstständigung nicht ins Leere laufen zu lassen.

40 Vgl. Siegel, Stadionverbote und andere polizeirechtliche Maßnahmen zur Eindämmung von Gewalt in Fußballstadien, in NJW 2013, $1035 \mathrm{ff}$.

41 Vgl. Breucker, Präventivmaßnahmen gegen reisende Hooligans, in: NJW 2004, 1631 ff.; Schucht, Die polizei- und ordnungsrechtliche Meldeauflage: Standortbestimmung und dogmatische Ausrichtung, NVwZ 2011, $709 \mathrm{ff}$.

42 Vgl. Breucker, (Fn. 41) 1632.

43 Vgl. EGMR, NVwZ 2014, $43 \mathrm{ff}$.

44 Vgl. EGMR, NVwZ 2014, 43 Rn. 70.

45 Vgl. Heidebach, Der polizeirechtliche Präventivgewahrsam auf konventionsrechtlichem Prüfstand, NVwZ 2014, 554 ff. (557).

46 Vgl. Verleih, (Fn. 35) 2014; Arbeitsgemeinschaft Fananwälte, (Fn. 35). 


\section{Zur materialistischen Kritik polizeilicher Repression}

Den Ausgangspunkt einer Kritik polizeilicher Verselbstständigung bildet eine staatstheoretische Reflexion über die Konstitution des modernen, bürgerlichen Staates. Deshalb sollen zunächst die Prämissen materialistischer Staatstheorie erläutert werden, um diese spezifische Form des Denkens über den Staat einzuführen. Hierbei wird der Fokus auf die spezifische Genese von Staatsapparaten gelegt. Anschließend wird die Polizei als Staatsapparat aus dieser Perspektive rekonstruiert. Zuletzt wird der hieraus gewonnene Analyserahmen genutzt, um die zu Anfang aufgeworfenen Beispiele polizeilicher Repression staatstheoretisch zu interpretieren.

\section{Materialistische Staatstheorie}

Die klassische Staatstheorie setzt sich nicht grundlegend kritisch mit ihrem Gegenstand auseinander, sondern setzt den Staat als zu legitimen Entscheidungen fähige und gegenüber gesellschaftlichen Konflikten neutrale Instanz. Dabei basiert die Begründung des Staates auf einem Gedankenexperiment: Thomas Hobbes als Vordenker säkularisierter Staatlichkeit erkennt die Notwendigkeit zur Einsetzung eines mit allumfassenden Rechten ausgestatteten Staates aufgrund eines von ihm selbst fingierten Naturzustandes, in dem die menschliche Natur als ursprünglich ,egoistisch“ und „feindselig“ vorausgesetzt wird. ${ }^{47}$ Die an Hobbes anschließende theoretische Weiterentwicklung dieses Konzeptes (durch vor allem Locke, Montesquieu und Rousseau) und die bahnbrechenden Revolutionen in Frankreich und Nordamerika „demokratisieren“ den Staat, indem seine Gewalt auf verschiedene Instanzen aufgespalten wurde. Es entstehen Parlamente, Gerichte und Verwaltungsbehörden, die sich gegenseitig kontrollieren sollen. Aber die Befugnis des Staates zu strafen, Gewalt auszuüben und seine Bürger_innen zu disziplinieren, wird nicht in Frage gestellt. Der Staat, so die Erzählung, basiere gerade auf dem demokratisch herausgebildeten „Volkswillen.“ Selbst Renate Mayntz als klassische Vertreterin der Staatsforschung in Deutschland, erkennt in einem solchen Zugang eine „relative Herrschaftsblindheit“".48 Lediglich die Theorietraditionen von Max Weber und die materialistische Staatstheorie würden einen herrschaftskritischen Ansatz verfolgen, der mit seiner Betonung der Herrschaftsanalyse aber nicht ,in den theoretischen mainstream" passt. ${ }^{49}$

Demgegenüber geht die materialistische Staatstheorie davon aus, dass die Strukturprinzipien, welche die Konstitutionalisierung des Staates bedingen, der kapitalistischen Vergesellschaftung entsprechen. So formuliert Joachim Hirsch: „Der Staat ist die Gestalt, die das politische Gemeinwesen unter den im Kapitalismus herrschenden gesellschaftlichen Bedingungen annimmt. Nicht nur der ökonomische, sondern auch der politische Zusammenhang der durch Konkurrenz und Klassenantagonismen geprägten

47 Eine Kritik klassischer Souveränitätstheorien findet sich bei: Loick, Kritik der Souveränität, Frankfurt am Main 2012.

48 Vgl. Mayntz, Zur Selektivität der steuerungstheoretischen Perspektive, dies. (Hrsg.), Über Governance. Institutionen und Prozesse politischer Regelung, Frankfurt am Main 2009, $29 \mathrm{ff}$. (33).

49 Ebd., 33. 
Gesellschaft manifestiert sich in einer von ihr getrennten und ihr äußerlich gegenübertretenden Instanz: Das gesellschaftlich Allgemeine erscheint als das von der Gesellschaft Abgesonderte. " ${ }^{50}$ Der Staat ist damit eine von der Gesellschaft und insbesondere der Ökonomie getrennte Institution, aber nur eine relativ autonome Institution, wie es Nicos Poulantzas formuliert. ${ }^{51}$ Zwar ist der Staat eine Institution, die von der Existenz kapitalistischer Produktionsverhältnisse abhängt, aber er ist gegenüber einzelnen politischen wie ökonomischen Fraktionen innerhalb der Gesellschaft autonom, um verschiedene Interessen zur Kohäsion zu bringen. Der Staat muss diese Kohäsion vollziehen, da kapitalistische Gesellschaften von grundsätzlich nicht auflösbaren Konflikten durchzogen und deshalb strukturell krisenhaft sind. Aber er kann durch seine Besonderung kein reines Abbild der Herrschaftsverhältnisse sein. „Er ist keine geschlossene Einheit, sondern ein Kampffeld. Gleichzeitig ist seine relative Einheitlichkeit Voraussetzung einer einigermaßen konsistenten Politik und damit der gesellschaftlichen Reproduktion. (...) Die Besonderung oder relative Autonomie des Staates ist somit immer das Ergebnis von durch seine eigene Materialität geformten Auseinandersetzungen und Kämpfen."52 Gesellschaftliche Widersprüche drücken sich deshalb ,institutionell in konkurrierenden Knoten, Machtnetzen und Entscheidungszentren innerhalb von staatlichen Apparaten aus, die jeweils Prämissen folgen."53

Dementsprechend ist auch die Gewaltenteilung des modernen Staates zu verstehen. ${ }^{54}$ Wenngleich die klassische Staatstheorie die Gewaltenteilung als demokratische Pazifizierung der im Staat angelegten Gewalt ansieht, ist die materialistische Staatstheorie demgegenüber skeptischer. Für Alex Demirovic stellt die Gewaltenteilung kein genuines Prinzip einer gegenseitigen Kontrolle der Gewalten dar, sondern eine „Verteilung der Macht der verschiedenen Fraktionen der herrschenden Klassen auf die Staatsapparate. "55 Die Gewaltenteilung fungiere als strategisches Moment staatlicher Herrschaftsausübung und ermögliche gerade ,verselbstständigte bürokratische Vorgänge“. 56

\section{Die Genese von Staatsapparaten}

Mit den Erkenntnissen materialistischer Staatstheorie wird also die Annahme vertreten, dass sich mit dem Staat keine monolithische Institution herausbildet, sondern ein Ensemble verschiedener Staatsapparate mit je eigenen Logiken, Interessen und Selektivitäten für gesellschaftliche Interventionen. So wird überhaupt erst die Möglichkeit theoretisch reflektierbar, dass unterschiedliche Staatsapparate je gegenläufige Strategien und

50 Hirsch, Materialistische Staatstheorie, Hamburg 2005, 26.

51 Vgl. Poulantzas, Staatstheorie, Hamburg 2002 (1978), 47 ff., 158 ff., 166 ff.

52 Hirsch/Kannankulam,/Poulantzas und Formanalyse. Zum Verhältnis zweier Ansätze materialistischer Staatstheorie, Bretthauer, u.a. (Hrsg.): Poulantzas lesen. Zur Aktualität marxistischer Staatstheorie, Hamburg 2006, 65 ff. (79).

53 Poulantzas, (Fn. 51), 167.

54 Vgl. Fisahn, Legitimation des Gewaltmonopols, KritV 01/2011, 3-17.

55 Demirovic, Rätedemokratie oder das Ende der Politik, Prokla 02/2009, $181 \mathrm{ff}$ (193).

56 Ebd., 193. 
Logiken verfolgen. Die Polizei als Teil der Exekutive und die Gerichte als Teile der Judikative können sich konfliktorisch gegenübertreten.

In Teilen der materialistischen Staatstheorie bleibt jedoch offen, welche Logiken sich auf welche Art und Weise in den Staatsapparaten manifestieren. Zur Überbrückung dieser Lücke kann das Staatstechnik-Theorem von Günter Frankenberg herangezogen werden. Er versucht die Ursprünge staatlicher Herrschaft zu ergründen und fragt angesichts der aktuellen Sicherheitsdebatte welche Vorstellungen staatlicher Macht verhandelt werden. Sein Begriff der Staatstechnik umfasst „die Gesamtheit der Verfahrensweisen, Normen und Prinzipien, Wissensformen und Kompetenzen, Berechnungen, Strategien und Taktiken, die von Akteuren und Institutionen operativ umgesetzt werden. ${ }^{\text {"57 }}$ Er identifiziert unterschiedliche Methoden, wie Staatstechnik von Institutionen umgesetzt wird. Die Methode Hobbes wird vor allem von „konservativen Staatslehrern und Sicherheitspolitikern“ genutzt, um ,politische Herrschaft und Ordnung vor den gefährlichen Elementen und überschießenden, ungezügelten Initiativen aus der Bürgerschaft in Schutz nehmen zu lassen." ${ }^{\circ 8}$ Es handelt sich um eine Methode, die staatliches Handeln vorrangig an dem Begriff der Sicherheit ausrichtet - der ehemalige Innenminister Hans-Peter Friedrich (CSU) bezeichnete die Sicherheit sogar als „,Supergrundrecht ${ }^{4}{ }^{59}$ Sicherheit ist der genuine Begriff der Polizei, ${ }^{60}$ ein Staatsapparat der vorrangig nicht den Bürgerrechten, sondern dem Schutz der staatlichen Ordnung verpflichtet ist. Es handelt sich um das ,Interesse des Staates an sich selbst ${ }^{\text {“ }}{ }^{61}$

Demgegenüber führt die Methode Locke „die Elemente des liberalen Paradigmas“62 ein, die Vorrangstellung der Exekutive in der Methode Hobbes wird abgelöst durch die „legislative Methode als Leitfunktion.“63 In Verteidigung der rechtsstaatlichen Methode konstatiert Frankenberg, dass die aktuellen Sicherheitsdebatten die Methode Hobbes reaktualisieren und zu einer Normalisierung von Sicherheit führen würden. ${ }^{64}$

Die Rolle des Rechts nimmt in dieser Konstellation nach Ingeborg Maus einen Doppelcharakter an, sowohl ,inhaltliche Ermächtigungen an das Staatshandeln auszusprechen und zugleich durch die Rechtsform zu begrenzen. “65 Das Recht soll demnach die Staatsapparate programmieren und ist damit der genuine Moment von Kohäsion, um die konfligierenden Logiken innerhalb der Staatsapparate zusammen zu bringen. Die Programmierungsfunktion des Rechts nimmt aber beständig ab, je entformalisierter das Recht wird. ${ }^{66}$ Durch die Ausweitung polizeilicher Generalklauseln nimmt das Recht eine Struktur an, die „die Staatsapparate immer weniger bindet“, eine Tendenz, „die in die gegenwärtig herrschende Verrechtlichung selbst eingebaut ist." ${ }^{\text {"67 }}$

57 Frankenberg, Staatstechnik. Perspektiven auf Rechtsstaat und Ausnahmezustand, Frankfurt am Main 2010, 17.

58 Ebd., 26.

59 Vgl. die Aussage von Hans-Peter Friedrich nach einer Sondersitzung des Parlamentarischen Kontrollgremiums zur NSA-Affäre im Juli 2013.

60 Marx, Zur Judenfrage, MEW 1, Berlin 1973 (1843), 347-370 (365).

61 Offe, Strukturprobleme des kapitalistischen Staates, Frankfurt am Main 2006 (1973), 130.

62 Frankenberg, (Fn. 57), 27.

63 Ebd., 29.

64 Ebd., 39.

65 Maus, Rechtstheorie und politische Theorie im Industriekapitalismus, München 1986, 315.

66 Ebd., 279.

67 Ebd., $279 \mathrm{f}$. 


\section{Die Polizei als Aufhebung der Gewaltenteilung}

Denn die spezifische Herausbildung und strukturelle Verankerung der Polizei im Staat, enthält eine illiberale Valenz: „Die zentrale Aufgabe (staatlicher) Aufrechterhaltung schreibt dem staatlichen Handeln die Suspension des Rechts der Möglichkeit nach immer schon ein."68 Diese Möglichkeit sieht Walter Benjamin in der spezifischen Absonderung der Polizei vom Recht, wenn er schreibt, ,dass die Zwecke der Polizeigewalt mit denen des übrigen Rechts stets identisch oder auch nur verbunden wären, (...) ist durchaus unwahr." 69 In dieser Bestimmung liegt ein Paradox. Denn es ist gerade die Polizei, die dem Recht Materialität verleiht. Keine Richter_innen stehen während einer Demonstration auf der Straße, sondern es ist die Polizei, die gegenüber den Bürger_innen als Repräsentantin des Rechts auftritt. Deshalb dürfe die Polizei nach dem Verständnis moderner Gesellschaften , keinen eigenen Inhalt haben, sie muss sich auf den Vollzug eines von anderer Seite determinierten Willens beschränken. “70

Diesem Argument klassischer Souveränitätstheorien stellt Benjamin das Argument entgegen, dass die Verunreinigung des Verhältnisses zwischen Zweck und Mittel hinsichtlich der Polizei gerade zu einer vom Recht losgelösten Verselbstständigung führe. Denn obschon die Polizei zur Exekutive gezählt wird, erwachsen ihr auch legislative und judikative Befugnisse: Die Polizei ist „rechtssetzende - denn deren charakteristischste Funktion ist ja nicht die Promulgation von Gesetzen, sondern jedweder Erlaß, den sie mit Rechtsanspruch ergehen läßt -, und sie ist rechtserhaltende, weil sie sich jenen Zwecken zur Verfügung stellt. (...) die Polizei (greift aber auch) in zahllosen Fällen ein, wo keine klare Rechtslage vorliegt, wenn sie nicht ohne jegliche Beziehung auf Rechtszwecke den Bürger als brutale Belästigung durch das von Verordnungen geregelte Leben begleitet oder ihn schlichtweg überwacht. ${ }^{\text {771 }}$ Die Polizei ist als Vollzugsorgan also exekutiv tätig, ihr kommt durch die Existenz von Generalklauseln die Möglichkeit zu, Recht zu setzen und sie ist judikativ tätig, indem sie in zahllosen alltäglichen Situationen allein entscheidet wie das Recht anzuwenden ist. Es ist diese strukturelle Aufhebung der Gewaltenteilung im Apparat der Polizei, der die Polizei nicht zu „einem Agent des Rechts, sondern gerade des Rechtsbruchs“ macht. ${ }^{72}$ Unabhängig davon obliegt es konkreten gesellschaftlichen Auseinandersetzungen darüber zu verhandeln, wie weit diese Entgrenzung der Polizei reichen kann.

\section{Polizeiliche Repression als Entgrenzung vom Recht}

Die von Benjamin diagnostizierte Aufhebung der Gewaltenteilung im Staatsapparat der Polizei verdeutlicht sich am Beispiel der zu Anfang einer juridischen Kritik unterzogenen Momente polizeilicher Repression.

68 Wallat, Die Herrschaft des Gesetzes und ihre Suspension. Ein Beitrag zur politischen Philosophie des Rechts(staates), in: Elbe u.a. (Hrsg.), Anonyme Herrschaft. Zur Struktur moderner Machtverhältnisse, Münster 2012, 112 ff. (164).

69 Benjamin, Zur Kritik der Gewalt und andere Aufsätze, Frankfurt am Main 1965 (1921), 44.

70 Loick, (Fn. 47), 183.

71 Benjamin, (Fn. 69), 44.

72 Loick, (Fn. 47), 183. 
Zunächst zu Blockupy. Sowohl während der Aktionstage 2012 als auch 2013 handelte die Polizei als Akteur, der die Abwesenheit rechtsstaatlicher Prinzipien erzeugte und eigenständige Interessen verfolgte. Ein Artikel aus der FAZ liefert ein anschauliches Psychogramm der Frankfurter Polizei. Der Ausgangspunkt ist ein, vermutlich durch Autonome verletzter Polizist auf der M31-Demonstration zwei Monate vor den Blockupy-Aktionen: „Die Frankfurter Polizei ist seit der 'M31'-Demonstration traumatisiert. Bis ins Mark getroffen von der eigenen Unfähigkeit linksextremistische Gewalt in diesem Ausmaß zu verhindern. Mehr noch als die Geschäftsleute, Banker und andere Bürger wurde die Polizei an jenem Tag selbst zum Opfer. So empfindet sie es zumindest." ${ }^{\text {"73 Die }}$ Polizei ist in dieser Darstellung kein reines Vollzugsorgan des staatlichen Interesses, sondern ein eigener Akteur, der auf Angriffe eigenmächtig reagiert. Aus diesem „Trauma der Polizei“" ist das rabiate Vorgehen gegen die Demonstrationen in Frankfurt zu erklären. Der FAZ-Artikel verdeutlicht, ,wie sehr die (Beamten, M.P.) darunter litten, dass auch sie als Staatsmacht, in ihren Befugnissen beschränkt sind. "74 Also suchte die Polizei gemeinsam mit dem ebenfalls auf Linie stehenden Ordnungsamt nach Möglichkeiten dieser Begrenzung zu entfliehen, um ihr Interesse, eine Verhinderung der Demonstrationen, zu ermöglichen. Hatte man es 2012 noch geschafft die Gerichte von den Gefahrenprognosen zu überzeugen und ein Totalverbot von Versammlungen durchgesetzt, scheiterte man mit diesem Ansinnen 2013. Zu sehr war die Stadt aus der Sicht der Polizei darum bemüht dem Blockupy-Bündnis entgegen zu kommen. Deshalb agierte man am Tag der Großdemonstration 2013 als ,Judikative der Straße“ und ersetzte die Erlaubnis des Verwaltungsgerichtes durch eine eigene Gefahrenprognose vor Ort - ,in den dabei entstandenen Räumen des Ausnahmerechts waren die machtaufschiebenden Potentiale des Rechts faktisch ausgehebelt. ${ }^{\prime 75}$ Der zuständige richterliche Notdienst sah sich zu keiner Kontrolle der Exekutive genötigt und vertraute alleine der Gefahrenprognose der Polizei. Es interessierte die Polizei nicht, ob sie mit diesen Maßnahmen das Grundrecht auf Versammlungsfreiheit missachtete, sondern es war gerade ihre Intention dieses nicht zur Geltung kommen zu lassen.

Bei den Gefahrengebieten in Hamburg wiederholt sich dieses Moment polizeilicher Entgrenzung auf andere Weise. Hier ist die Polizei nicht judikativ tätig, sondern tritt im Sinne Benjamins legislativ auf. Die vollkommen unzureichend bestimmte Generalklausel des hamburgischen Polizeirechts ermöglicht es ein Gefahrengebiet nach dem Gusto der Polizei auszurufen. Dies verweist auf die bereits von Ingeborg Maus problematisierte Funktion von Generalklauseln. Eine inhaltliche Ausfüllung dieser Normen wird nicht von der Legislative, sondern von der sie umzusetzenden Exekutive vorgenommen. Die Entgrenzung der Polizei ist nur vor diesem Hintergrund möglich, indem sich die Legislative eigenständig ihrer Befugnisse entledigt. Das Ergebnis ist eine ebenso von Moritz Assall diagnostizierte ,polizeiliche Verselbstständigung, die mitnichten Ausnahmezustand, sondern latenter Charakter der Polizei im bürgerlichen Rechtsstaat ist. ${ }^{\text {"76 }}$ Zum Teil ermöglichen Generalklauseln sogar die Etablierung vollkommen neuer

73 Iskandar, Die verwundete Polizei, Frankfurter Allgemeine Sonntagszeitung vom 9.6.2013, 9.

74 Ebd., 9.

75 Petzold,/Pichl, Räume des Ausnahmerechts: Staatliche Raumproduktionen in der Krise am Beispiel der Blockupy-Aktionstage 2012, KrimJ 03/2013, 211 ff. (224).

76 Assall, (Fn. 24) 79. 
Staatsapparate, wie beispielsweise die Hessische Stadtpolizei, deren Existenz auf verfassungsrechtlich fragwürdigem Boden gründet. ${ }^{77}$

Die konkrete Betrachtung der Gefahrengebiete in Hamburg zeigt aber, dass Benjamin mit seinem Argument bezüglich der legislativen Funktion der Polizei etwas zu weit geht und den eigentlichen Kern des Problems nicht hinreichend trifft. Im Anschluss an Maus besteht das Problem nicht darin, dass die Polizei Recht setzen würde. Benjamin verwechselt faktisches exekutivisches Handeln mit formeller Rechtssetzung. Die Polizei nutzt vielmehr Lücken des Rechtes aus und füllt diese mit ihren eigenen Zwecken. Generalklauseln ermöglichen es der Polizei ihre Gefahrenprognosen, ihre Einschätzungen und ihre Interessen in der Logik des Rechts zu verallgemeinern. Diese Handhabung des Rechts durch die Polizei ist deutlich subtiler und hintergründiger, als wenn die Polizei selbst als Gesetzgeber auftreten würde. Indem die Polizei ihre Zwecke praktisch mit den Worten des Rechts verhüllt, sind ihre Maßnahmen der Kritik schwer zugänglich. Der Rechtsbruch durch die Polizei vollzieht sich innerhalb des Rechts und nicht außerhalb der juridischen Logik.

Im Hinblick auf die Repressionen gegen Fußballfans ist die Polizei der vorrangige Akteur, der die Kriminalisierung dieser Szene vorantreibt. Angesichts von stetig sinkenden Gewalttaten und polizeilichen Aufgabenfeldern, konstruierte die Polizei den „Feindbild Fußballfan“, um dem drohenden Personalabbau - dem Interesse an dem eigenen Staatsapparat - entgegen zu wirken. ${ }^{78}$ Die Polizeigewerkschaften sind dabei die Pioniere in dem Versuch immer neue Repressionsmaßnahmen im Stadion auszutesten. Erstaunlich ehrlich sagte Rainer Wendt, Vorsitzender der Deutschen Polizeigewerkschaft (DPolG), in einem Interview nach der Verabschiedung des neuen Fußball-Sicherheitspapiers ,,man dürfe jetzt auch einmal sprachlich abrüsten. “79 Er gibt damit zu, dass die Polizeigewerkschaften eine Skandalisierung betreiben, um Repressionen gegen Fußballfans in der Bevölkerung zu legitimieren und zu normalisieren. Die Polizei beteiligt sich im Bereich Fußball selbst an der Gesetzgebung, sie verfolgt nicht vorrangig das Interesse mögliche Gewalttaten zu verhindern, sondern versucht sich ein neues Aufgabenfeld zu erschließen. Zugleich weiß die Polizei, dass es für mit dem Stigma „Gewalttäter" behaftete Fußballfans praktisch keine Lobby gibt. Sie kann dementsprechend das Stadion als Laboratorium nutzen, um neue Repressionsmaßnahmen auszutesten. Aufenthaltsverbote und Ausreiseverbote, die gegenüber Fußballfans eingesetzt werden, können dann bei politischen Demonstrationen zur Anwendung gelangen. Die Polizei vertraut auf die Normalisierung dieser Methoden in der Öffentlichkeit.

\section{Epilog}

Die Empörung über polizeiliche Rechtsübertretungen ist groß. In den letzten Jahren häufen sich in vielen Zeitungen kritische Kommentare über polizeiliches Handeln, es kommt zu Demonstrationen - auch außerhalb des linken Spektrums - gegen Polizeige-

77 Vgl. Fenger/Tohidipur/Tuchscherer, Die hessische Stadtpolizei, in: LKRZ 2013, $451 \mathrm{ff}$.

78 Vgl. Verleih, (Fn. 35).

79 Vgl. die Sky Sondersendung ab Minute 42:00, https://www.youtube.com/watch? $v=r B-$ qf9u6FDJU (zuletzt abgerufen am 9. August 2014). 
walt und immer wieder steht die Frage im Raum: Warum handelt die Polizei gegen das Recht? Die Antwort einer materialistischen Kritik an der Polizei gibt hierauf die Antwort: weil die Form des Rechts ihr strukturell die Möglichkeit verleiht, sich im Rahmen des Rechts vom Recht zu entgrenzen. Die spezifische Form der bürgerlichen Gewaltenteilung und die Möglichkeit, dass Staatsapparate je spezifische Logiken ausbilden, ermöglicht es der Polizei fernab von Parlaments- oder Gerichtsentscheidungen autonom zu entscheiden, wie und welche Maßnahmen sie zur Aufrechterhaltung der öffentlichen Sicherheit anwendet. Mit einer rein juridischen Kritik, die darauf abzielt die Polizei der rechtlichen Einhegung zuzuführen, wäre deshalb wenig gewonnen. Dies bedeutet nicht, dass die juridische Kritik suspendiert gehört. Die Einführung einer individuellen Kennzeichnung von Polizeibeamt_innen oder die Einsetzung einer unabhängigen Kontrollund Beschwerdestelle wären zu begrüßende Instrumente, um Betroffenen von Polizeigewalt überhaupt die Möglichkeit zu geben, juristisch gegen die Polizei vorzugehen. Ein Gerichtsprozess ist aber darauf ausgelegt, nicht die Polizei als Ganzes der Kritik zuzuführen, sondern befasst sich mit Einzelmaßnahmen von einzelnen Beamt innen. Gerade die Tatsache, dass sich die Polizei von der Einhegung des Rechts emanzipieren kann, verweist auf die strukturellen Probleme polizeilicher Gewalt. Um endlich über diesen Zustand hinaus zu kommen, bedürfte es eines gesellschaftlichen Projektes das generell die benjaminische Frage reaktualisiert, ob „Gewalt überhaupt, als Prinzip, selbst als Mittel zu gerechten Zwecken sittlich sei. “80

80 Benjamin, (Fn. 69), 29. 